\title{
Stretching a self-interacting semiflexible polymer
}

\author{
A. $\operatorname{RosA}^{1}$, D. Marenduzzo ${ }^{2}$ and S. Kumar ${ }^{1,3}$ \\ 1 Max-Planck Institut für Physik komplexer Systeme, NöthnitzerStrasse 38, 01187 Dres- \\ den, Germany \\ 2 School of Physics, SUPA, University of Edinburgh, Edinburgh EH9 3JZ, UK \\ 3 Department of Physics, Banaras Hindu University, Varanasi 221005, India
}

PACS. 82.35.Lr - Physical properties of polymers.

PACS. 87.15.-v - Biomolecules: structure and physical properties.

\begin{abstract}
We present results from three-dimensional off-lattice Monte-Carlo simulations to investigate the stretching response of a semi-flexible polymer subjected to self-attractive interactions. We employ the quasi-static approximation and consider both the fixed elongation and the fixed force ensemble, which can equally well be reproduced in experiments nowadays. The force versus elongation curves are in general non-monotonic, and the quantity and height of peaks increase with decreasing temperature, and with increasing stiffness. We finally compute the variation of unfolding force with temperature. Our results should be relevant for stretching experimental studies, and for more refined theoretical modeling, taking non-equilibrium and kinetic effects into account.
\end{abstract}

Introduction. - In recent years, the elastic properties of bio-polymers like proteins and DNA have been thoroughly investigated experimentally, thanks to the rapid development of single-molecule techniques, e.g. soft micro-needles [1], atomic force microscopes (AFMs) [2] and optical tweezers [3]. A major motivation for these studies comes from the possibility to enhance our quantitative understanding of the physical and biochemical properties of biomolecules. For instance, monitoring the applied tension at the ends of a macromolecule offers an insight into the molecular forces acting among its constituents. The detailed scenario emerging from the experimental force-elongation curves has called for the validating of a number of theoretical models previously introduced in polymer physics. The Freely Jointed Chain (FJC) [4] and the Worm-like Chain (WLC) models [5] are arguably the simplest and most widely known ones. These models predict markebly different shapes for the force versus elongation curves for a polymer, notably for intermediate to large forces $[4,6-8]$. Briefly, evidence has shown that the FJC model is appropriate for flexible polymers like polyethylene, while using the WLC model is crucial when fitting elasticity data for double-stranded (ds) DNA and other semi-flexible polymers and proteins.

However, neither of these models take into account of the excluded volume, and their validity is further limited to cases in which self-interactions between distinct polymer segments are negligible. This is appropriate in the strong-force regime, or far from the $\theta$ point (or folding temperature) for a self-attractive chain, such as a DNA molecule with multivalent counterions 


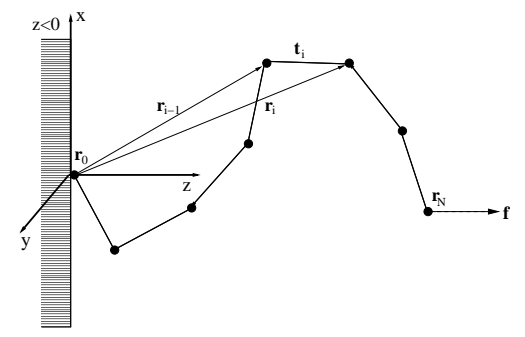

Fig. 1 - Schematics of the studied polymer system. The chain is attached to one end at $\boldsymbol{r}_{0}$, taken as the origin of the frame, whilst all the configurations with a monomer at $z<0$ are forbidden. The stretching force is directed along the $z$-direction. Each bond between consecutive beads has length $b=1$. Consecutive vectors $\boldsymbol{r}_{i-1}$ and $\boldsymbol{r}_{i}$ and their difference $\boldsymbol{t}_{i}=\boldsymbol{r}_{i}-\boldsymbol{r}_{i-1}$ are also shown.

[9] or a protein. Nonetheless, it would be of major interest to characterize with similarly coarse grained models and good accuracy what goes on whenever self-interactions cannot be neglected. In such cases we know that the stretching response is much more complicated, as has been found with on and off lattice simulations in 2 and 3 dimensions [10-13]. Even though an increasing amount of work has now been published on the subject (see also [14-16]), there is still a wealth of outstanding questions, which call for detailed theoretical calculations not relying on any mean field or large force approximation but carefully taking into account the interactions among different monomers. Thus, here we set out to compute the force versus elongation curves for a semi-flexible polymer subject to self-attractive interactions, using 3-dimensional Monte-Carlo simulations. Our model and results should be of relevance to stretching experiments with condensed DNA or other semi-flexible polymers in poor solvent condition [17]. We show that, in the constant distance ensemble, which may be attained by soft microneedles or laser tweezer experiments, the force changes in a non-monotonic way even neglecting kinetic effects, and a multi-peak pattern is in general observed at low temperature. It is important to have a reference calculation which predicts the shape of these curves in quasiequilibrium, in order to precisely estimate kinetic effects in the experiments or in molecular or Langevin dynamics simulations $[18,19]$. Furthermore it should be possible to perform single molecule experiments at very low pulling speed, to test the results we predict here. Our simulations show that stiffness greatly enhances the height and number of peaks in the force versus elongation curves. Such peaks are ultimately due to non-convexity regions in the free energy as a function of extension along the pulling force. We also sketch a phase diagram featuring the temperature dependence of the unfolding force on temperature, which can be experimentally measured to test whether the model we tackle here may yield a faithful representation of DNA biophysics over a range of temperatures, or if other details are needed for that, as in the case of e.g. DNA unzipping [20].

Model. - Our algorithm simulates a self-interacting chain of $N+1$ beads connected by rigid bonds of length $b=1$, and stretched at one end by a constant force $\boldsymbol{f}$. Since in most experiments the polymer is attached to a substrate, we have treated the plane $z=0$ as an impenetrable wall (see ref. [2]). In fig. 1, we show the coordinate axis as well as the schematic of set-up used in our model system. The Hamiltonian $\mathcal{H}$ for this system is given by

$$
\beta \mathcal{H}=\beta \mathcal{H}(f)=-\beta K_{s} \sum_{i=1}^{N-1} \boldsymbol{t}_{i} \cdot \boldsymbol{t}_{i+1}+\beta \sum_{i=0}^{N-2} \sum_{j \geq i+2}^{N} V\left(\left|\boldsymbol{r}_{i}-\boldsymbol{r}_{j}\right|\right)-\beta \boldsymbol{f} \cdot\left(\boldsymbol{r}_{N}-\boldsymbol{r}_{0}\right),
$$


where we have explicitly included the dependence on the force $\boldsymbol{f}$ - whose modulus we call $f$. In eq. (1), $\beta=1 / K_{B} T$, where $K_{B}$ denotes the Boltzmann constant (here, taken to be 1 ) and $T$ the temperature. The first term in the Hamiltonian is the Kratky-Porod term to describe semiflexible polymers [4], and $K_{s}$ is the stiffness constant or elastic modulus (it has the dimension of an energy times a time scale), while $\boldsymbol{t}_{i}=\boldsymbol{r}_{i+1}-\boldsymbol{r}_{i}(i=1, \ldots, N)$ is the vector joining two consecutive beads. The second term corresponds to the self-interaction potential between non-consecutive beads, which is of the form of a square-well as described below

$$
V(r)= \begin{cases}\infty & \text { if } r<r_{0} \\ -V_{0} & \text { if } r_{0}<r<r_{1} \\ 0 & \text { otherwise }\end{cases}
$$

with $V_{0}=1, r_{0}=0.8$ and $r_{1}=1.2$. The potential is chosen in such a way that, for $f=0$ and relatively low $T$, the polymer is found in a compact state. We note that the transition to the compact state occurs at a stiffness dependent temperature. Moreover, the square-well potential has been chosen for the computational efficiency.

From standard thermodynamical relations, we know that the partition function of the system described by eq. (1) is given by

$$
\mathcal{Z}=\mathcal{Z}(f)=\int \prod_{i=1}^{N} d \boldsymbol{r}_{i} e^{-\beta \mathcal{H}(f)}
$$

and the average extension $z$ along the stretching force is

$$
\langle z\rangle=-\frac{1}{\beta} \frac{\partial}{\partial f} \log \mathcal{Z}(f) .
$$

In eq. (4), $\langle\cdot\rangle$ means the ensemble average with respect to the Boltzmann distribution. Another important quantity of experimental interest is the end-to-end distribution function or Green function [4], $\mathcal{G}_{f}(\boldsymbol{r})$, mathematically defined as

$$
\mathcal{G}_{f}(\boldsymbol{r})=\left\langle\delta\left(\boldsymbol{r}_{N}-\boldsymbol{r}_{0}-\boldsymbol{r}\right)\right\rangle=\frac{1}{\mathcal{Z}(f)} \int \prod_{i=1}^{N} d \boldsymbol{r}_{i} e^{-\beta \mathcal{H}(f)} \delta\left(\boldsymbol{r}_{N}-\boldsymbol{r}_{0}-\boldsymbol{r}\right),
$$

where $\delta$ denotes Dirac's delta. After integrating this function yields the partition function, it contains all the information which is needed to compute the thermal averages we are interested in. Of particular interest to us is the number of polymer conformations whose projection of the end-to-end distance along $f$ is $z$. The corresponding reduced Green function $G_{f}(z)$ is given by

$$
G_{f}(z)=\int d x d y \mathcal{G}_{f}(\boldsymbol{r}) \equiv G_{0}(z) e^{-\beta f z} .
$$

From $G_{0}(z)$, one can readily extract the average $\langle f\rangle$ at fixed distance $z$, see e.g. ref. [21],

$$
\langle f\rangle=-\frac{1}{\beta} \frac{\partial}{\partial z} \log G_{0}(z) .
$$

It is known that the two ensembles - fixed distance and fixed force - are equivalent only in the thermodynamical limit $[12,22]$. It will either be obvious from the context or specified in each case which ensemble we use in the following. Hereafter, we shall make use of the scaled elongation $\zeta=z / N$. To investigate the stretching response of the polymer system under consideration, we adopted the reweighted histograms technique [23,24], which has revealed to be very powerful in studying phase transition in interacting systems. In present case this has allowed us to obtain precise estimates for $\langle z\rangle$ and $\langle f\rangle$. Note that all simulations presented here were performed with $N=45$. Different values of $N$ yield qualitatively similar results. 


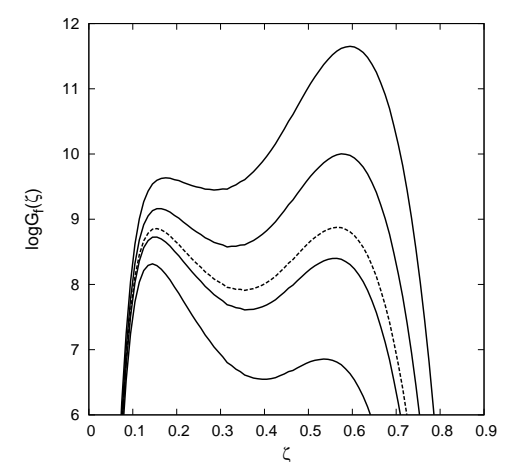

Fig. 2 - Plots at different stretching forces of the logarithm of the Green function $\log G_{f}(\zeta)$ versus the scaled elongation $\zeta=z / N$ (see eq. 6) at $T=0.8$, with $K_{s}=0$ and $N=45$. The solid lines represent the plots for $f=2.7,2.75,2.8,2.85$ (from bottom to top). For clarity, we have also plotted the same function around the transition force $f_{t} \simeq 2.765$ (dashed line).

Results and discussion. - To fix ideas, let us first analyze the limit case of a selfinteracting flexible chain $\left(K_{s}=0\right.$ in eq. (1)). Fig. 2 shows the logarithm of the Green function $\log G_{f}(\zeta)$ as a function of the scaled elongation $\zeta=z / N$, for various values of $f$ (see caption). The temperature is $T=0.8$ (which is below the $\theta$ point for all $K_{s}$ considered here). From fig. 2 the bi-modal nature of the free energy is apparent. This means that the unfolding transition is first order, in agreement with the literature [13]. (We note that the order of this transition changes in 2 dimensions, where it is continuous $[10,11,13])$. In fig. 2 and in the rest of the paper, the statistical error in $\log G_{f}(\zeta)[24]$ has been found to be at most $2 \%$.

Let us now consider how this scenario is modified for $K_{s} \neq 0$. In fig. 3 (left panel) we have plotted the force vs extension curves, in the fixed elongation ensemble [see eq. (7)] for $K_{s}=0,1.2$ and 2 , where $T=0.8$ as of fig. 2 . The curves display a non-monotonic behaviour, in agreement with what has been recently found in molecular dynamics $[25,26]$ and theoretical calculations on the stretching response of a homopolymer. Our curves typically display first a maximum and then a minimum below full stretching. This means that the system has to overcome a free energy barrier before going into the unfolded state (fig. 3). The nonmonotonicity is formally a consequence of the double well shape attained by $\log G_{f}(\zeta)$ near criticality, observed in Fig. 2. Such a behaviour is in sharp contrast with the corresponding curves calculated in the fixed force ensemble, shown as continuous curves in Fig. 3 (left panel). There is thus a strong inequivalence between the two ensembles, as in the fixed force ensemble, due to the thermodynamical averaging, the oscillations are completely washed out. Moreover, stiffness enhances the sharpness of the transition. In particular, this feature is evident if we plot $\log G_{f}(\zeta)$ vs. $\zeta$ for the various $K_{s}$ used and in the vicinity of the corresponding transition forces (fig. 3, right panel). As anticipated, the height of the free energy barrier - hence the height of the peaks in the force vs elongation curves - is enhanced by stiffness.

We now turn to the analysis of the stretching behaviour at fixed stiffness for different temperature. A different scenario appears here. In fig. 4 we have shown the results for $K_{s}=0$ (left panel) and $K_{s}=2$ (right panel) at $T=0.8$ and 0.4. Fig. 4 demonstrates that the polymer stiffness and the attracting potential compete each other to give rise to a multi-peak behaviour in the force versus elongation characteristic curves. This can also be appreciated upon inspection of fig. 5 (left panel), where $\log G_{f}(\zeta)$ vs. $\zeta$ is plotted, for $T=0.8$ and $T=0.4$, in the vicinity of the transition forces. The multi-peak behaviour is similar to the 

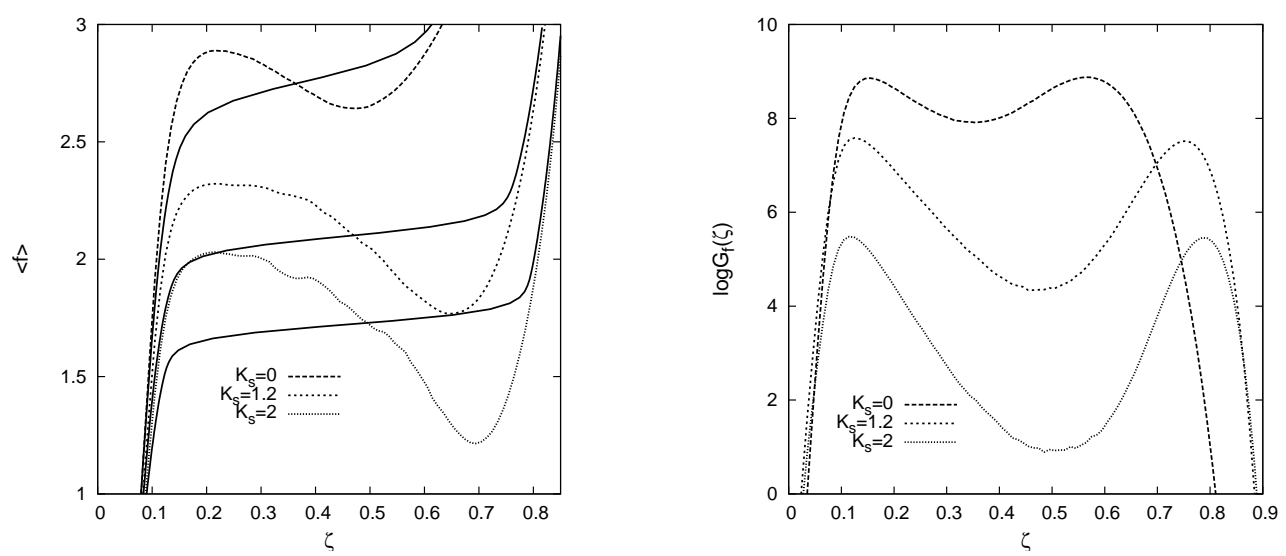

Fig. 3 - (Left panel) $\langle f\rangle$ vs. the scaled elongation $\zeta=z / N$ at $T=0.8$, with $N=45$ and for different values of the stiffness parameter $K_{s}$. From top to bottom, $K_{s}=0, K_{s}=1.2$ and $K_{s}=2$. The solid curves are the corresponding plots in the fixed force ensemble. Differences between the two ensembles are apparent. (Right panel) The sharpness of the transition in the fixed force ensemble increases with polymer stiffness. The depth of the potential barrier for $\log G_{f}(\zeta)$ (shown for the same values of $K_{s}$ and in the proximity of the corresponding transition forces) displays a similar behaviour. Note that the plots for $K_{s}=1.2$ and $K_{s}=2$ have been shifted up of a constant to help the comparison.

one observed in refs. $[12,22]$ for semi-flexible polymers and polymethylene chains. In all these cases the peak pattern was usually ascending, whereas in the present one it displays a more irregular behaviour. It would be interesting to compare experimental measurement of force versus elongation characteristic curves for semiflexible polymers in a poor solvent, to clarify this issue. We also note that in a related calculation on the square lattice it was found that the probability distribution of the end-to-end distance showed oscillations for sufficiently stiff chains [27], which shows that free energy profile of such system is quite rough. Moreover, peaks
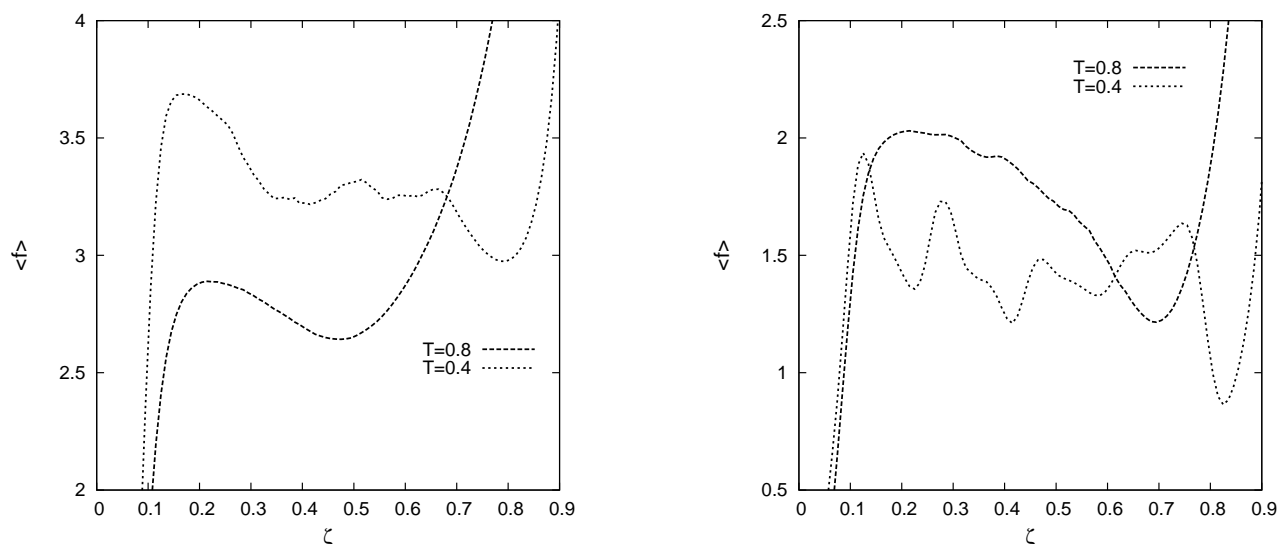

Fig. 4 - (Left panel) Plot of $\langle f\rangle$ vs. $\zeta$ for $K_{s}=0$, with $N=45$, and at temperatures $T=0.8$ (dashed line) and $T=0.4$ (dotted line). (Right panel) $\langle f\rangle$ vs. $\zeta$ for $K_{s}=2$, with $N=45$ and at temperatures $T=0.8$ (dashed line) and $T=0.4$ (dotted line). Here, in particular, the pronounced multi-peak behaviour in the low- $T$ limit is evident. 

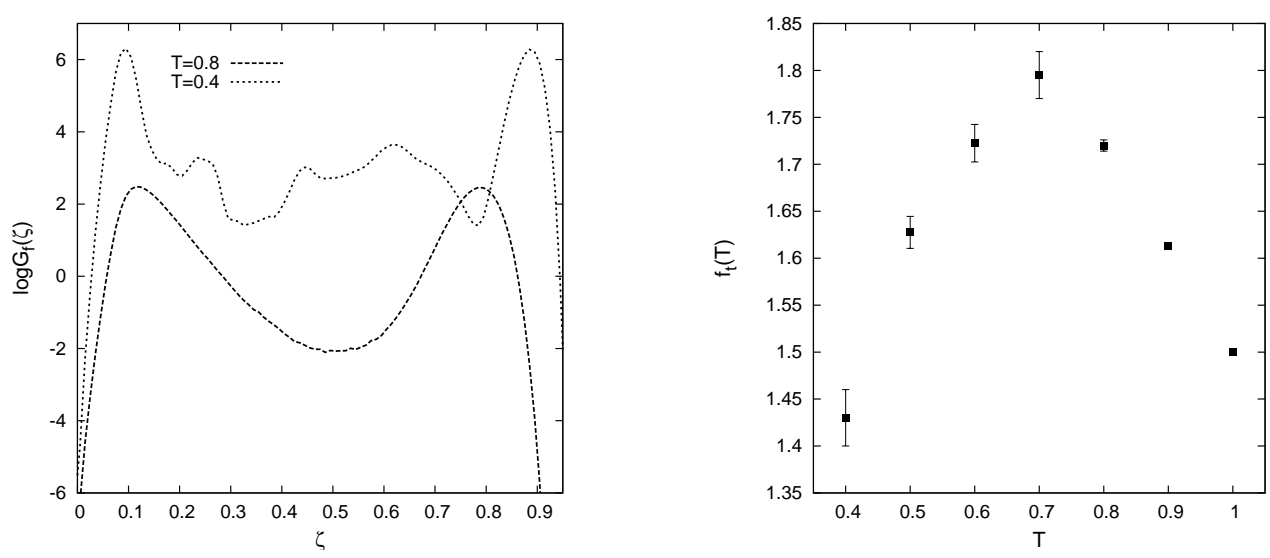

Fig. 5 - (Left panel) Plot of $\log G_{f}(\zeta)$ as a function of $\zeta$ for $T=0.8$ and $T=0.4$ and in the proximity of the corresponding transition forces. (Right panel) Phase diagram showing the transition force $f_{t}(T)$ vs. the temperature $T$.

in the force versus elongation curves have also been found in Langevin dynamics simulations of the stretching of a flexible polymer in a poor solvent [26]. It would be nice to couple simulations as in [26] to our Monte-Carlo quasi-static calculations to pinpoint in a clean and quantitative way the magnitude of kinetic and hydrodynamic effects in the stretching response of a semi-flexible polymer in computer simulations. In simulations performed here the minimum of the force curves just before full stretching corresponds to the unraveling transition described in [26]. In fig. 6 we have plotted three configurations obtained from a simulation performed at $f=1.175$ and $T=0.4$, that exemplifies the presence of many states around the transition force. The observed multi-peak behaviour is physically due to this fact. In particular the central snapshot reproduce a ball-and-chain configuration which has also been seen in refs. $[25,26]$.

Fig. 5 (right panel) finally shows how the transition force $f_{t}(T)$ for a semi-flexible chain with $K_{s}=2$ varies with temperature $T$ between 0.4 and 0.8 . The transition force is defined for each temperature $T$, as the force at which the derivative with respect to the applied force of $\zeta$ has a maximum. The reproduced values are averages over five independent simulations
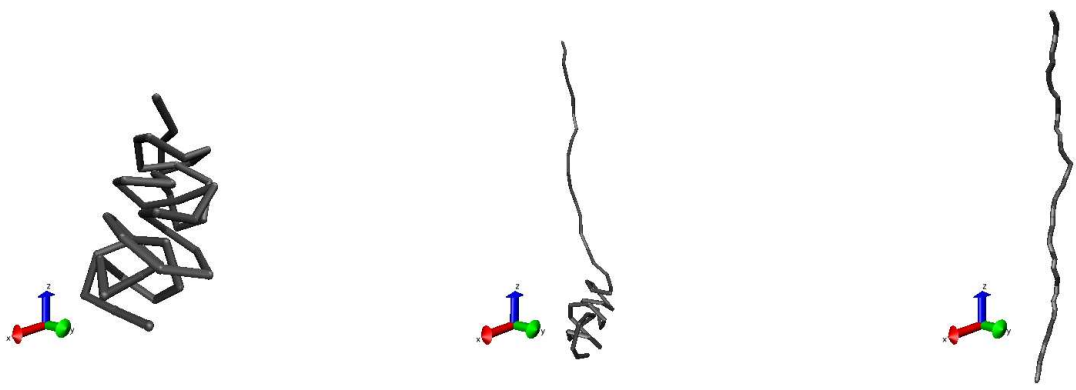

Fig. 6 - Snapshots of three configurations obtained at force $f=1.175$ and $T=0.4$. The left configuration looks like an elongated globule along the $z$-direction (blue arrow). The central snapshot represents a ball-and-chain configuration $[25,26]$, while the right one is the full stretched chain. 
and error bars correspond to standard deviations. This is a different procedure than the one employed for fig. 4 (right panel) and fig. 5 (left panel), where the curves for $T=0.4$ were obtained reweighting together the histograms coming from all five simulations.

Conclusions. - In conclusion, we have presented here results obtained by coupling fully 3dimensional off-lattice Monte-Carlo simulations to a standard histogram reweighting technique to investigate the stretching response of a semi-flexible polymer of varying stiffness, subjected to self-attractive interactions. The present model of a semi-flexible polymer in a poor solvent should be of relevance to experiments with DNA in solutions with multivalent counterions or to proteins or heteropolymers. We have worked out the force versus elongation curves in the fixed elongation and fixed force ensembles, under the quasi-static assumption that the quantities of interest are not affected by kinetic effects such as the speed of stretching etc. While this situation may be achieved in experiments by sufficiently slow stretching, we anticipate that an important use of our results and of our methodological framework should be that of providing a reference for the estimate of force versus elongation curves in quasiequilibrium situation. Then, a comparison with molecular or Langevin dynamic calculations such as those reported in refs. [25,26] will allow a clean quantitative determination of kinetic, hydrodynamics or non-equilibrium effects in stretching real and numerical experiments, which would be of high relevance to the field of single molecule experiments.

Acknowledgments. - We thank N. Becker for a careful reading of the manuscript.

\section{REFERENCES}

[1] Kishino A. And Yanagida T., Nature, 34 (1988) 74.

[2] Rief M., Clausen-Schaumann H. and Gaub H. E., Nat. Struct. Biol., 6 (1999) 346.

[3] Bockelmann U., et al., Biophys. J., 82 (2002) 1537.

[4] Dor M. and Edwards S. F., Theory of Polymer Dynamics (Oxford University Press, Oxford) 1988.

[5] Kratky O. and Porod G., Rec. Trav. Chim., 68 (1949) 1106.

[6] Marko J. F. and Siggia E. D., Macromolecules, 28 (1995) 8759.

[7] Ha B.Y. and Thirumalai D., J. Chem. Phys., 106 (1997) 4243.

[8] Rosa A., Hoang T. X., Marenduzzo D. and Maritan A., Macromolecules, 36 (2003) 10095.

[9] Baumann C. G., et al., Biophys. J., 78 (2000) 1965.

[10] Marenduzzo D., Maritan A., Rosa A. and Seno F., Phys. Rev. Lett., 90 (2003) 088301.

[11] Rosa A., Marenduzzo D., Maritan A. and Seno F., Phys. Rev. E, 67 (2003) 041802.

[12] Marenduzzo D., Maritan A., Rosa A. and Seno F., Eur. Phys. J. E, 15 (2004) 83.

[13] Grassberger P. and Hsu H. P., Phys. Rev. E, 65 (2002) 031807.

[14] Halperin A. and Zhulina E. B., Europhys. Lett., 15 (1991) 417.

[15] Wada H., Murayama Y. and Sano M., Phys. Rev. E, 72 (2005) 041803.

[16] Geissler P.L. and Shakhnovich E.I., Phys. Rev. E, 65 (2002) 056110.

[17] P.-G. DE Gennes, Scaling Concepts in Polymer Physics (Cornell University Press, Ithaca) 1979.

[18] Cieplak M., Hoang T.-X. and Robbins M.O., Proteins, 49 (2002) 104.

[19] Cieplak M., Hoang T.-X. and Robbins M.O., Phys. Rev. E, 69 (2004) 011912.

[20] Danilowicz C. et al., Phys. Rev. Lett., 93 (2004) 078101.

[21] Flory P. J., Statistical Mechanics of Polymer Chains (Interscience, New York) 1969.

[22] Zemanova M. and Bleha T., Macromol. Theory Simul., 14 (2005) 596.

[23] Ferrenberg A. M. and Swendsen R. H., Phys. Rev. Lett., 61 (1988) 2635.

[24] Ferrenberg A. M. and Swendsen R. H., Phys. Rev. Lett., 63 (1989) 1195.

[25] Cieplak M., Hoang T. X. and Robbins M. O., Phys. Rev. E, 70 (2004) 011917.

[26] Cooke I. R. and Williams D. R. M., Europhys. Lett., 64 (2003) 267.

[27] Kumar S. and Giri D., Phys. Rev. E, 72 (2005) 052901. 